\title{
Osteoporosis knowledge, beliefs, and calcium intake of college students: Utilization of the health belief model
}

\author{
Ellen Edmonds ${ }^{1}$, Lori W. Turner ${ }^{2}$, Stuart L. Usdan ${ }^{2}$ \\ ${ }^{1}$ Department of Health Science, University of Central Arkansas, Conway, USA; eedmonds@uca.edu \\ ${ }^{2}$ Department of Health Science, University of Alabama, Tuscaloosa, USA; lwturner@ches.ua.edu, susdan@ches.ua.edu
}

Received 12 October 2011; revised 15 November 2011; accepted 22 December 2011

\begin{abstract}
Objective: The purpose of this study was to examine the relationships between osteoporosis knowledge, beliefs and calcium intake among college students. This study also examined perceived susceptibility, severity, benefits, barriers and self-efficacy related to osteoporosis prevention. Participants: Seven hundred and ninety two $(n=$ 792) men and women ages 17 - 31 of all ethnicities at a mid-western regional university in the US participated in the study. Methods: The Osteoporosis Knowledge Test, Osteoporosis Health Belief Scale, and Osteoporosis Preventing Behaviors Survey were utilized. Each of these tools were previously validated and found reliable. Correlation and multiple regression analyses were completed. Results: Participants did not perceive themselves as susceptible to osteoporosis and perceived minimal barriers to calcium intake. Their knowledge was minimal concerning alternate sources of calcium. Conclusions: Prevention programs should aim to increase osteoporosis knowledge of risk factors and osteoprotective behaviors and to decrease high-risk behaviors during college years when behavior changes can have the strongest impact on bone health.
\end{abstract}

Keywords: Osteoporosis; Calcium; Prevention; Health Belief Model; Bone Health

\section{INTRODUCTION}

Osteoporosis is a skeletal disorder characterized by compromised bone strength, predisposing an individual to an increase risk of fracture [1]. The World Health Organization adds to the above definition and defines osteoporosis as a bone mineral density value more than 2.5 standard deviations below the mean for normal young white women [2]. The most common sites of fractures are at the spine, wrist, and hip. In individuals with osteoporosis, the bands or plates of bone become thin, porous and weakened [3]. When porous bone fractures, it shatters in pieces that often cannot be reassembled.

By 2020, one in two Americans over age fifty will have or be at risk for developing osteoporosis [3]. The Surgeon General has identified bone health as a critical component to the overall health and quality of life of Americans [3]. Approximately ten million Americans have osteoporosis [4]. Of these, eight million are women and two million are men. In addition to these two million men, another thirtyfour million men have low bone mass [5]. Osteoporosis characteristically begins early in life when corrective action might slow down disease progression [6]. Women are at two to three times higher risk than men because of the rapid bone loss at menopause due to sharp declines in estrogen production [7].

Fractures are the most devastating problem facing people with osteoporosis [8]. In 1995, osteoporotic fractures contributed to more than half a million hospitalizations and over 800,000 emergency room visits [3]. Hip fractures are the most disabling type of fracture in people with osteoporosis. More than one in four individuals suffering a hip fracture becomes disabled during the following year. Nearly one in five requires long-term nursing home care. In 1995, 140,000 persons were admitted to nursing homes due to hip fractures [3].

Deaths related to bone disease usually have indirect causes. Fractures and their related complications can trigger a downward spiral in health. Approximately 20\% of hip fracture patients die within a year of the fracture [8]. Hip fractures were listed as the cause of death on 12,661 death certificates in 1999 [3]. Nine out of one hundred women with a hip fracture will die as a result of the fracture [9]. Fractures resulting from osteoporosis can lead to pain, height loss, inability to stand, and inability to walk [10]. Hip fractures are the most disabling type of fracture [11] and usually result in two million person-years of permanent disability [12]. 
The expense of fractures is costly. Currently, the annual direct care expenditures for osteoporotic fractures are $\$ 12.2$ - \$17.9 billion per year [13]. Hip fractures are the most costly of all fractures, representing \$11.3 billion of the total direct expenditure of osteoporosis [14,15].

Some of the risk factors for osteoporosis are genetics, smoking and alcohol abuse, poor nutrient intake, calcium and vitamin D deficiency, and decrease in sex hormone production [3]. Corrective action must be taken slow down bone loss and prevent osteoporosis [7]. Prevention of bone disease should begin at birth and continues throughout life. According to the Department of Health and Human Services' Surgeon General's Report, the challenge is to address the lack of awareness of bone disease in the public and even among health care professionals [3]. Many health care professionals do not understand the magnitude of the problem or the ways in which bone disease can be prevented and treated. One of the common misconceptions is that osteoporosis is an inevitable part of aging and that it is limited to older White women [16,17]. This incorrect view has delayed prevention and treatment in young and middle-aged women, men and minority women. In addition, misconceptions regarding osteoporosis have delayed educational efforts among youth.

To address issues related to lack of awareness, use of health behavior theories may increasing the effectiveness of assessing osteoporosis knowledge and developing education efforts. The Health Belief Model (HBM) can be used to gain an understanding of health behaviors and reasons for non-compliance related to osteoprotective behaviors [18-20]. This model can assess perceived susceptibility, severity, benefits, barriers, and self efficacy [20,21].

The purpose of this study was to determine the level of osteoporosis knowledge, beliefs, and calcium intake among college students. In addition, this study examined the constructs of the Health Belief Model (HBM), perceived susceptibility, severity, benefits, barriers, and health motivation, related to osteoporosis prevention.

\section{METHODS}

Institutional Review Board approval at the educational institution was obtained and each student was provided an informed consent letter prior to responding to the survey. Three surveys were disseminated to participating college students. Permission to use the Osteoporosis Health Belief Scale (OHBS), the Osteoporosis Knowledge Test (OKT) and the Osteoporosis Preventing Behaviors Survey (OPBS) was obtained.

The OHBS [22] is a 42-item instrument consisting of seven subscales addressing health beliefs. The subscales address susceptibility, severity, benefits to exercise, benefits to calcium intake, barriers to exercise, barriers to calcium intake, and health motivation. Each item was rated using a 5 point Likert scale with 1 = strongly disagree, 2
= disagree, 3 = neutral, 4 = agree, and 5 = strongly agree . Agree or strongly agree are correct responses.

Scores had a possible range of 42 to 210 for the total health belief score and a possible range of 6 to 30 for each subscale score. Preliminary testing of women revealed the test-retest reliability for the total instrument as 0.90 and the subscale test-retest reliabilities ranged from 0.52 to 0.84 [22]. Concurrent validity was established through assessment of calcium and exercise behaviors along with the Health Belief Model (HBM) instrument.

The OKT [22] is a twenty-four item tool consisting of two subscales addressing exercise (16 items) and calcium intake (17 items). The survey includes 9 items that address knowledge of overall osteoporosis risk factors. Each item is rated by the participant using $\mathrm{ML}=$ more likely, $\mathrm{LL}=$ less likely, NT = neutral, and DK = don't know. Scores for calcium had a possible range of 0 to 17 . Through preliminary testing of the instrument on a sample population of women, the test-retest reliability coefficient for OKT calcium was 0.72 and OKT exercise was 0.69 [23].

The Osteoporosis Preventing Behaviors Survey (OPBS) [24] is a 39-item self-report descriptive survey that addresses osteoporosis preventing behaviors involving the categories of activities/exercise, dietary intake of calcium, and other risk factors (smoking, alcohol use, use of hormonal therapy, use of non-hormonal therapy and other medications that affect bone density) and included other demographic items.

Questions related to calcium intake include 4 items. Each item in questions 1 - 3 is rated by the subject by using 1 = none per week, 2 = one per week, 3 = two per week, 4 = three per week, $5=$ four per week, $6=$ five per week, 7 = six per week, 8 = one per day, $9=$ two per day, $10=$ three per day. Question 4 is rated as $1=$ no and $2=$ yes to answer the question of intake of a calcium supplement. For analysis of the behavior calcium intake, three groupings were established [25]. Less than four servings a week indicated inadequate intake. Five servings a week to one serving a day were moderate intake. Two to three servings a day were considered adequate intake.

To determine internal consistency, Cronbach alpha was utilized [27]. A reliability coefficient of 0.70 or higher is considered acceptable in most social science research situations [28]. All reliability coefficients are within the acceptable range except the osteoporosis knowledge test which yielded a coefficient of 0.69 .

After collection of the questionnaires, missing data variables were coded as 99. Next, variables were coded with a numerical code. A test for outliers was also conducted. Three extreme outliers were discovered and were recoded. Descriptive statistics were generated on all variables. A Pearson correlation was used to determine the degree of relationship between variables.

The dependent variable for the multiple regression analy- 
sis was calcium intake (a combined variable of milk, cheese, yogurt, and Ca supplement intake). The independent variables were osteoporosis knowledge, attitudes, perceived susceptibility, perceived severity, perceived benefits, perceived barriers, gender, ethnicity, family history, age, calcium intake, and physical activity.

After determining which independent variables were significant through a bivariate correlation analysis, a multiple regression analysis was completed. Multicolinearity of each independent variable was assessed by examining the correlations and associations between the independent variables.

\section{RESULTS}

A response rate of $96 \%$ was obtained. This study included 792 men and women who were enrolled in a course in the Health Science Department during the spring 2009 semester. The majority of these students were majors in the health science department, and the remaining students were collected from a general education course in the department. Originally, the questionnaire was given to 825 students; however, 33 were eliminated due to incompletion of the demographic questions. The majority of the students were nineteen to twenty-one years of age (58.5\%), with a mean age of 20.6 years. The study sample was comprised of more female (57.3\%) than male students and more White (64.5\%) than any other ethnicity. Table 1 presents the demographics of the sample.

Overall, participants did not perceive themselves to be susceptible to osteoporosis and strongly disagreed with the statement "family history makes it more likely" (35.4\%). However, they found themselves neutral to the statement “your chances of getting osteoporosis are high" (31.2\%). Approximately 45\% (45.3) agreed that it would be very serious to get osteoporosis, $35.5 \%$ also agreed with the statement "osteoporosis would be very costly", and 33.5\% agreed with the statement "the thought of having osteoporosis scares them”. However, participants did not agree with the statement “osteoporosis would be crippling” (36.1\%) and did not agree with the statement "I get depressed when thinking of osteoporosis” (31.8\%).

All participants indicated agreement with statements of exercise and calcium intake as protective toward osteoporosis, and students disagreed with statements designed to assess perceived barriers to physical activity or calcium intake. The participants were neutral (39.0\%) on the barrier statement "calcium rich foods have too much cholesterol".

Although many participants disagreed with the statement "you have a regular health check-up” (29.2\%), overall there was agreement with statements designed to assess health motivation.

Since this study was examining each separate construct, the six questions per construct were computed into a new variable named for each construct. Table 2 presents the mean and standard deviation for each health belief construct.

For the construct of perceived susceptibility, the possible score is 6 - 30 with a greater number representing greater susceptibility for osteoporosis. The sample had a mean of 13.64 and standard deviation of 5.09 for the construct susceptibility which indicates low perceived susceptibility for osteoporosis.

Table 1. Demographics of the study sample $(n=792)$.

\begin{tabular}{lll}
\hline Demographic & & $\mathrm{n}(\%)$ \\
\hline & $16-18$ & $121(15.3 \%)$ \\
Age (years) & $22-24$ & $463(58.5 \%)$ \\
& $25-27$ & $152(19.2 \%)$ \\
& $28-30$ & $24(3 \%)$ \\
Gender & 31 or over & $13(1.6 \%)$ \\
& Male & $19(2.4 \%)$ \\
& Female & $338(42.7 \%)$ \\
Ethnicity & White & $454(57.3 \%)$ \\
& African American & $209(26.4 \%)$ \\
& Hispanic American & $16(2 \%)$ \\
& Other & $56(7 \%)$ \\
\hline
\end{tabular}

Table 2. Osteoporosis health belief scale-total constructs $(n=792)$.

\begin{tabular}{|c|c|c|}
\hline Constructs & Mean (SD) possible score 6 - 30 & Interpretation related to osteoporosis \\
\hline Perceived susceptibility & $13.64( \pm 5.09)$ & Low perceived susceptibility \\
\hline Perceived severity & $17.34( \pm 4.37)$ & Moderate perceived seriousness \\
\hline Perceived benefits of exercise & $23.23( \pm 5.33)$ & High perceived benefits \\
\hline Perceived benefits of calcium intake & $22.26( \pm 4.61)$ & High perceived benefits \\
\hline Perceived barriers to exercise & $24.27( \pm 4.62)$ & Low perceived barriers \\
\hline Perceived barriers to calcium intake & $22.82( \pm 4.57)$ & Low perceived barriers \\
\hline Perceived health motivation & $19.87( \pm 4.34)$ & Positive view of health \\
\hline
\end{tabular}


For the construct of perceived seriousness, the possible score is 6 - 30 with a greater number representing a perception of osteoporosis being serious. The sample had a mean of 17.34 and standard deviation of 4.37 for the construct seriousness, which indicates a view of osteoporosis as moderately serious.

For the construct of perceived benefits for both physical activity and calcium intake, the possible score is 6 30 , with a greater number representing a positive view of exercise and calcium intake. The mean for the construct perceived benefits of physical activity was 23.23 with a standard deviation of 5.33. The mean for perceived benefits of calcium intake was 22.26 with a standard deviation of 4.61. These scores represent a positive view of both constructs.

For both perceived barriers to physical activity and calcium intake, the possible score is 6 - 30 with a higher number representing fewer barriers. The mean for perceived barriers to physical activity is 24.27 (standard deviation of 4.62). The mean for perceived barriers to calcium intake is 22.83 (standard deviation of 4.57). These scores show that participants had few reported barriers to either physical activity or calcium intake.

The last construct of the Health Belief Model was perceived health motivation. The possible score is 6 - 30 with a higher number representing higher motivation for health. The mean is 19.87 with a standard deviation of 4.34 which indicates a positive motivation for health just above the mean.

Most participants incorrectly thought neither having ovaries surgically removed (34.4\%) nor being a white woman with fair skin (38.4\%) increased the risk of acquiring osteoporosis. They also did not view having big bones (38.9\%) as being a protective factor to osteoporosis.

Correctly, participants identified jogging/running for exercise $(76.8 \%)$ and aerobic dancing (77.4\%) as physical activity which reduces chances of osteoporosis. Incorrectly, a majority of participants identified swimming (50.8\%) as being a protective factor for osteoporosis.

Correctly, the participants thought it will take three or more days of physical activity a week (80.3\%) and a minimum of twenty to thirty minutes (75.9\%) a day of physical activity to protect against osteoporosis.

In regards to foods which are good sources of calcium, the participants correctly recognized cheese (89.0\%), broccoli (50.8\%), yogurt (87.1\%), and ice cream (66.2\%). As stated above, participants correctly identified traditional sources of calcium; however, they were not able to recognize alternate sources of calcium such as canned sardines (23.5\%).

Participants knew the recommended amount of milk is two or more glasses daily (45.6\%). However, they incorrectly identified the recommended amount of calcium intake for adults as $400 \mathrm{mg}$ - $600 \mathrm{mg}$ daily (39.8\%). Eleven percent recognized the correct amount of calcium needed by adults as $800 \mathrm{mg}$ or more a day.

Table 3 represents the students' current calcium intake behaviors. The responses were divided into three categories: inadequate, moderate, and adequate. Inadequate represents a response of zero to four servings of calcium a week. Moderate represents a response of five servings of calcium a week to one serving of calcium a day. Adequate represents a response of two servings to three servings of calcium a day. The answers provided by the highest proportion of respondents are bolded for each of the statements.

There was also a positive correlation between calcium intake (a combined variable including all Ca sources listed above $)$ and health motivation $(\mathrm{r}=0.204, \mathrm{n}=790, \mathrm{p}=$ 0.000 ), with high levels of calcium intake associated with a high motivation to health. Calcium intake had a non-significant, low correlation to perceived barriers to physical activity $(r=0.041, n=787, p=0.217)$. However, calcium intake also had a low correlation to perceived benefits of calcium intake that did not reach statistical significance $(\mathrm{r}=0.050, \mathrm{n}=788, \mathrm{p}=0.159)$.

Multiple regression, as shown in Table 4, was used to assess the ability of six independent variables (ethnicity, health motivation, age, perceived barriers to physical activity, perceived barriers to calcium intake, and physical activity) to predict calcium intake. Preliminary analyses were conducted to ensure normality, multicollinearity, and homoscedasticity were not violated. However, as previously mentioned, calcium intake was not found to be linear.

After entry of the variables the total variance explained by the model was $12.2 \%, \mathrm{p}<0.05$. In the final model, all predictors were statistically significant, with ethnicity recording a higher beta value $(\beta=-0.173, \mathrm{p}<0.05)$ than other variables.

Table 3. Osteoporosis preventing behaviors survey $(\mathrm{n}=792)$.

\begin{tabular}{llc}
\hline \multicolumn{1}{c}{ Behavior } & & Percent \\
\hline \multirow{2}{*}{ Glasses of milk } & Inadequate & $62.5 \%$ \\
& Moderate & $24.1 \%$ \\
& Adequate & $13.1 \%$ \\
Eight-ounce servings of yogurt & Inadequate & $94.0 \%$ \\
& Moderate & $4.5 \%$ \\
Servings of cheese & Adequate & $1.4 \%$ \\
& Inadequate & $56.0 \%$ \\
& Moderate & $29.5 \%$ \\
& Adequate & $14.2 \%$ \\
\hline
\end{tabular}

Inadequate $=$ Less than 4 servings of calcium a week. Moderate $=5$ servings of calcium a week to 1 serving a day. Adequate $=2$ servings of calcium a day to 3 servings a day. Most frequent responses are bolded. 
Table 4. Summary of multiple regression analysis for variables predicting calcium intake.

\begin{tabular}{lccc}
\hline Variable & B & SE B & $\beta$ \\
\hline Barriers/PA & -0.193 & 0.048 & $-0.171^{*}$ \\
Ethnicity & -1.069 & 0.211 & $-0.173^{*}$ \\
MET-MIN & 8 & 0.008 & $0.162^{*}$ \\
Barriers/Ca & 0.191 & 0.046 & $0.168^{*}$ \\
Health Motivation & 0.186 & 0.043 & $0.154^{*}$ \\
Age & 0.428 & 0.183 & $0.080^{*}$ \\
\hline
\end{tabular}

R Square $=0.122 ;{ }^{*} \mathrm{p}<0.05$.

Internal consistency measures estimate how consistently individuals respond to the items within a scale. In social science research the cut-off is that alpha should be 0.70 or higher for a set of items to be considered a scale. All reliability coefficients are within the acceptable range except the osteoporosis knowledge test which yielded a coefficient of 0.69 .

\section{COMMENT}

Many studies linked with osteoporosis have spotlighted knowledge of and the health beliefs of individuals. The majority of studies observe white, pre and postmenopausal women, and few studies have examined men or multiple ethnicities.

Prevention is the most effective way to promote bone health. It is critically important to build and to maintain strong, healthy bones throughout life. To accomplish this, everyone should understand the basics of the prevention of osteoporosis. Calcium intake is essential in developing healthy bones [3]. Review of the literature exposed a consensus that most individuals are not engaging in preventative measures for osteoporosis.

Researchers are attempting to determine which variables lead some individuals to perform these behaviors and which variables hinder a person's performance. Knowledge of osteoporosis is limited in all populations, and even if an individual has knowledge of osteoporosis, it does not increase the likelihood he/she will engage in the preventative behaviors. Other important variables in possibly increasing behaviors that prevent osteoporosis are whether the person feels susceptible, whether the person feels osteoporosis is severe, the view of the benefits of calcium intake, the view of the barriers of calcium intake.

The HBM proposes that if individuals are to take osteoporosis prevention measures, they must feel susceptible to osteoporosis, believe that occurrence of osteoporosis would have a severe impact on their lives, and conclude that preventive measures are beneficial, outweighing any barriers involved in taking the actions [29]. Par- ticipants in this study did not feel susceptible to osteoporosis, and according to the HBM, they will not take preventative measures to ward off the disease. Questions examining the construct of perceived severity were answered with mixed results. Overall, they did not believe that osteoporosis would significantly affect their lives. In this study, the benefits of physical activity and calcium intake did outweigh the barriers. The study population had positive views of both physical activity and calcium intake and reported few barriers to both activities.

Lack of osteoporosis knowledge is well documented among both genders and different ethnicities. Prevention and treatment in controlling osteoporosis are effective; however, unless individuals are familiar with them and are able to make informed choices regarding available treatments, those measures are useless. Reports show knowledge concerning the risk of a lack of physical activity and low calcium intake [30]. They also determined the inability to identify risk factors of skipped menstrual periods and postmenopausal status [30]. This study produced similar results. The majority of participants were able to identify risks of a lack of physical activity and low calcium intake but were unable to identify the risks inherent in white women, the risks that come with the removal of ovaries, and the benefits to big-boned individuals.

Participants in the study seemed to possess knowledge that calcium plays a role in keeping bones strong. However, they were unable to correctly identify the recommended amount of calcium for adults. They were also unable to identify alternate sources of calcium.

One of the findings from this study was a lack of perceived susceptibility towards osteoporosis among college age students. This is consistent with findings that stated college females perceive breast cancer and heart disease as greater threats than osteoporosis [21]. Reports show that perceived susceptibility was considerably lower in young adults compared to older age groups [31], and it was determined that $50 \%$ of their study population believed osteoporosis was a minor health problem [32]. One possible explanation for young women not perceiving themselves as susceptible is the thought that osteoporosis is mainly an older woman's disease. It was concluded that $65 \%$ of women ages eighteen to twenty-five thought osteoporosis was a disease of women over seventy years of age [33].

Another finding was this population thought osteoporosis to be moderately severe. It was found that perceived severity was an important construct when determining noncompliance in preventative behavior [20]. In other words, the less severe a person believes osteoporosis to be, the less likely he/she is to participate in behaviors which shield him/her from osteoporosis. In an article [32], 53\% of women thought osteoporosis was a curable disease; this idea would therefore lower their perceived severity of the disease. 
A contradictory finding from this study is that a majority of the study population had low perceived barriers to calcium intake. These participants consumed inadequate amounts of calcium. This is in contrast to findings [34] who determined that perceived barriers were the most common factor impacting behavior. If perceived barriers were high, the population would not engage in preventative behaviors. However, this population's perceived barriers were low, typically meaning behaviors would be high; however, this population contradicts this rule by not acquiring the recommended amount of calcium.

Knowledge about osteoporosis is inconsistent in the study population, with a moderate understanding of the need to include calcium rich foods and an understanding of the forms of physical activity that could help prevent osteoporosis. However, in the physical activity questions, there was one choice response which was a more traditional type of exercise and the other choices were more everyday types of physical activity. This may have led the study population to choose the traditional exercise response without actually having knowledge of which enhanced the prevention of osteoporosis.

\section{STRENGTHS OF THE STUDY}

In addition to having a high response rate, the strengths of the study included high statistical power because of the large sample size. The data collection method was strengthened by the fact that only two researchers collected the data. The collection method was consistent in each class. The primary researcher inputted all data; therefore, it was controlled and closely monitored. Data entry verification was conducted preceding data analysis. This was carried out by verifying every ten entries were correct.

\section{LIMITATIONS}

The limitations related to this research project include its sampling method. Convenience sampling is selecting research participants on the basis of being accessible and convenient to the researcher [35]. This can be accomplished by using students in classes in a particular department [36].

One potential limitation to using a convenience sample is that a claim for representativeness of the population can not be made. Another limitation is the researcher's ability to generalize findings beyond the actual sample may be limited [36]. An additional limitation was the majority of the participants were majors in the health science department which may have led to a greater knowledge of this area due to their interest in health issues.

Participants tend to report what they believe the researcher expects to see or they wish to present themselves in a socially acceptable manner [37]. Another concern about such data centers on whether subjects are able to accurately recall past behaviors. Cognitive psychologists have warned that the human memory is fallible [38] and thus the reliability of self-reported data is tenuous on some items.

\section{CONCLUSIONS}

Young adults are heading in a direction that will add them to the national burden of osteoporosis. Coupled with a greater number of our population living longer, osteoporosis is becoming an increasing public health concern. Research indicates the importance of prevention in the fight against osteoporosis [3]. Prevention programs should aim to increase knowledge and to decrease risk factor behaviors in adolescence when bone health can be increased. This would help adolescents achieve a higher bone mass as a means of helping them prevent or delay the development of osteoporosis [39].

Although there are studies related to college students' nutritional habits, calcium intake, physical activity, and osteoporosis risk factors, studies addressing osteoporosis prevention among both genders and multiple ethnicities are limited. Evidence that this population lacks knowledge about osteoporosis risk factors, calcium intake, and physical activity related to bone health makes it important to provide educational opportunities in several venues to increase knowledge. The importance of this is supported by those who found that individuals with greater knowledge related to the importance of calcium intake and physical activity are more likely to participate in those behaviors than those who do not have this knowledge [30]. In providing health education one must go beyond knowledge alone, recognizing that knowledge alone does not translate to behavior [40]. However, without knowledge, behavior change is not informed, and individuals must be aware of osteoporosis before they can be expected to take preventive steps.

\section{REFERENCES}

[1] (2000) National Institue of Health Consensus Statement Online. Osteoporosis Prevention, Diagnosis, and Therapy, 17, 1-36.

[2] World Health Organization (2003) The burden of musculoskeletal conditions at the start of the new millennium, WHO Technical Report Series 919, World Health Organization, Geneva.

[3] US Department of Health and Human Services \& Centers for Disease Control and Prevention (2004) Bone health and osteoporosis: A report of the surgeon general, Atlanta.

[4] National Osteoporosis Foundation (2002) American's bone health: The state of osteoporosis and low bone mass in our nation. National Osteoporosis Foundation, Washington DC.

[5] National Osteoporosis Foundation (2008) National Osteoporosis Foundation-Osteoporosis facts. http://www.nof.org/osteoporosis/diseasefacts.htm\#cost 
[6] Seeman E. (2003) Invited review: Pathogenesis of osteoporosis. Journal of Applied Physiology, 95, 2142-2151.

[7] Riggs, B.L., Khosla, S. and Melton III, L.J. (2002) Sex steroids and the construction and conservation of the adult skeleton. Endocrine Reviews, 23, 279-302. doi:10.1210/er.23.3.279

[8] Leibson, C.L., Tosteson, A.N., Gabriel, S.E., Ransom, J.E. and Melton, L.J. (2002) Mortality, disability, and nursing home use for persons with and without hip fracture: A population-based study. Journal of American Geriatric Society, 50, 1644-1650. doi:10.1046/j.1532-5415.2002.50455.x

[9] Maganziner, J., Lydick, E., Hawkes, W., Fox, K.M., Zimmerman, S.I., Epstein, R.S. and Hebel, J.R. (1997) Excess mortality attributable to hip fracture in white women aged 70 years and older. American Journal of Public Health, 87, 1630-1636. doi:10.2105/AJPH.87.10.1630

[10] Salkeld, G., Cameron, I.D., Cumming, R.G., Easter, S., Seymour, J., Kurrle, S.E., et al. (2000) Quality of life related to fear of falling and hip fracture in older women: A time trade off study. British Medical Journal, 26, 292-298.

[11] Kanis, J.A. and Johnell, O. (1999) The burden of osteoporosis. Journal of Endocrinology Investment, 22, 583588.

[12] Chrischilles, E., Shireman, T. and Wallace, R. (1994) Costs and health effects of osteoporotic fractures. Bone, 15, 377-386. doi:10.1016/8756-3282(94)90813-3

[13] Tosteson, A.N.A. (1999) Economic impact of fractures. In: Orwoll, E.S., Eds., Osteoporosis in men: The effects of gender on skeletal health, Academic Press, San Diego, 15-27.

[14] Ray, N.F., Chan, J.K., Thamer, M. and Melton III, L.J. (1997) Medical expenditures for the treatment of osteoporotic fractures in the United States in 1995: Report from the National Osteoporosis Foundation. Journal of Bone Mineral Research, 12, 24-35. doi:10.1359/jbmr.1997.12.1.24

[15] Gabriel, S.E., Tosteson, A.N., Leibson, C.L., Crowson, C.S., Pond, G.R., Hammond, C.S. and Melton III, L.J. (2002) Direct medical costs attributable to osteoportic fractures. Osteoporosis International, 13, 323-333. doi:10.1007/s001980200033

[16] Mudano, A.S., Casebeer, L., Patino, F., Allison, J.J., Weissman, N.W., Kiefe, C.I., Person, S., Gilbert, D. and Saag, K.G. (2003) Racial disparities in osteoporosis prevention in a manged care population. Southern Medical Journal, 96, 445-451. doi:10.1097/01.SMJ.0000053918.93363.B0

[17] Qaseem, A., Snow, V., Shekelle, P., Hopkins, R., Forciea, M.A. and Owens, D.K. (2008) Screening for osteoporosis in men: A clinical practice guideline from the American college of physicians. Annals of Internal Medicine, 148, 680-684.

[18] Chan, M.F., Kwong, W.S., Zang, Y. and Wan, P.Y. (2007) Evaluation of an osteoporosis prevention education programme for young adults. Journal of Advanced Nursing,

\section{3, 270-285. doi:10.1111/j.1365-2648.2006.04091.x}

[19] Sedlak, C.A., Doheny, M.O. and Jones, S.L. (2000) Osteoporosis education programs: Changing knowledge and behaviors. Public Health Nursing, 17, 398-402. doi:10.1046/j.1525-1446.2000.00398.x

[20] Turner, L.W., Hunt, S.B., DiBrezzo, R. and Jones, C. (2004) Design and implementation of an osteoporosis prevention program using the health belief model. American Journal of Health Studies, 19, 115-121.

[21] Kasper, M.J., Garber, M. and Walsdorf, K. (2007) Young women's knowledge and beliefs about osteoporosis: Results from a cross-sectional survey of college females. American Journal of Health Education, 38, 186-193.

[22] Kim, K., Horan, M. and Gendler, P. (1991) Osteoporosis health belief scale. Presented at Sigma Theta Tau International Research Conference, Columbus.

[23] Kim, K., Horan, M. and Gendler, P. (1991) Osteoporosis knowledge test (Unpublished).

[24] Doheny, M. and Sedlak, C. (1995) Osteoporosis preventing behaviors survey (Unpublished).

[25] Sizer, F. and Whitney, E. (2008) Nutrition: Concepts and controversies. 11th Edition, Thompson-Wadsworth, Belmont.

[26] SPSS Inc. (2009) Statistical package for the social sciences. http://www.spss.com/

[27] Pallant, J. (2007) SPSS Survival manual. 3rd Edition, McGraw Hill, New York.

[28] Daniel, W.W. (1999) Biostatistics: A foundation for analysis in the health sciences. 7th Edition, John Wiley \& Sons, Inc., Hoboken.

[29] Rosenstock, I.M. (1974) The health belief model and preventive health behavior. Health Education Monographs, 2, 328-335.

[30] Kasper, M.J., Peterson, G.E. and Allegrante, J.P. (2001) The need for comprehensive educational osteoprosis prevention programs for young women: Results from a second osteoporosis prevention survey. Arthritis Care Research, 45, 28-34. doi:10.1002/1529-0131(200102)45:1<28::AID-ANR80>3 .0.CO;2-G

[31] Johnson, C.S., McLeod, W., Kennedy, L. and McLeod, K. (2008) Osteoporosis health beliefs among younger and older men and women. Health Education and Behavior, 35, 721-733. doi:10.1177/1090198107301331

[32] Drozdzowska, B., Pluskiewicz, W. and Skiba, W. (2004) Knowledge about osteoporosis in a cohort of Polish females: The influence of age, level of education and personal experiences. Osteoporosis International, 15, 645648. doi:10.1007/s00198-003-1581-2

[33] Hazavehei, S.M., Taghdisi, M.H. and Saidi, M. (2007) Application of the health belief model for osteoporosis prevention among middle school girl students, Garmsar, Iran. Education for Health, 20, 1-11.

[34] Wallace, L. (2002) Osteoporosis prevention in college women: Application of the expanded health belief model. American Journal of Health Behavior, 26, 163-172. doi:10.5993/AJHB.26.3.1 
[35] Baumgartner, T.A. and Hensley, L.D. (2006) Conducting and reading research in health and human performance. 4th Edition, McGraw-Hill, New York.

[36] Neutens, J.J. and Rubinson, L. (2002) Research techiques for health sciences. 3rd Edition, Benjamin-Cummings, San Francisco.

[37] Cook, T. and Campbell, D. (1979) Quasi-experimentation: Design and analysis issues. Houghton Mifflin Company, Boston.

[38] Schacter, D. (1999) The seven sins of memory: Insights from psychology and cognitive neuroscience. American
Psychology, 54, 182-203. doi:10.1037/0003-066X.54.3.182

[39] Kasper, M.J., Peterson, G.E., Allegrante, J.P., Galsworthy, T.D. and Gutin, B. (1994) Knowledge, beliefs, and behaviors among college women concerning the prevention of osteoporosis. Archives of Family Medicine, 3, 696-702. doi:10.1001/archfami.3.8.696

[40] Ribeiro, V., Blakeley, J. and Laryea, M.(2000) Women’s knowledge and practices regarding the prevention and treatment of osteoporosis. Health Care for Women International, 21, 347-353. doi:10.1080/073993300245195 